\title{
Téoros
}

Revue de recherche en tourisme

\section{Le tourisme hivernal des Québécois : de l'appel du sud au ski alpin}

\section{Roger Nadeau}

Volume 8, numéro 3, novembre 1989

Tourisme hivernal

URI : https://id.erudit.org/iderudit/1080304ar

DOI : https://doi.org/10.7202/1080304ar

Aller au sommaire du numéro

Éditeur(s)

Université du Québec à Montréal

ISSN

0712-8657 (imprimé)

1923-2705 (numérique)

Découvrir la revue

Citer cet article

Nadeau, R. (1989). Le tourisme hivernal des Québécois : de l'appel du sud au ski alpin. Téoros, 8(3), 3-7. https://doi.org/10.7202/1080304ar d'utilisation que vous pouvez consulter en ligne.

https://apropos.erudit.org/fr/usagers/politique-dutilisation/ 


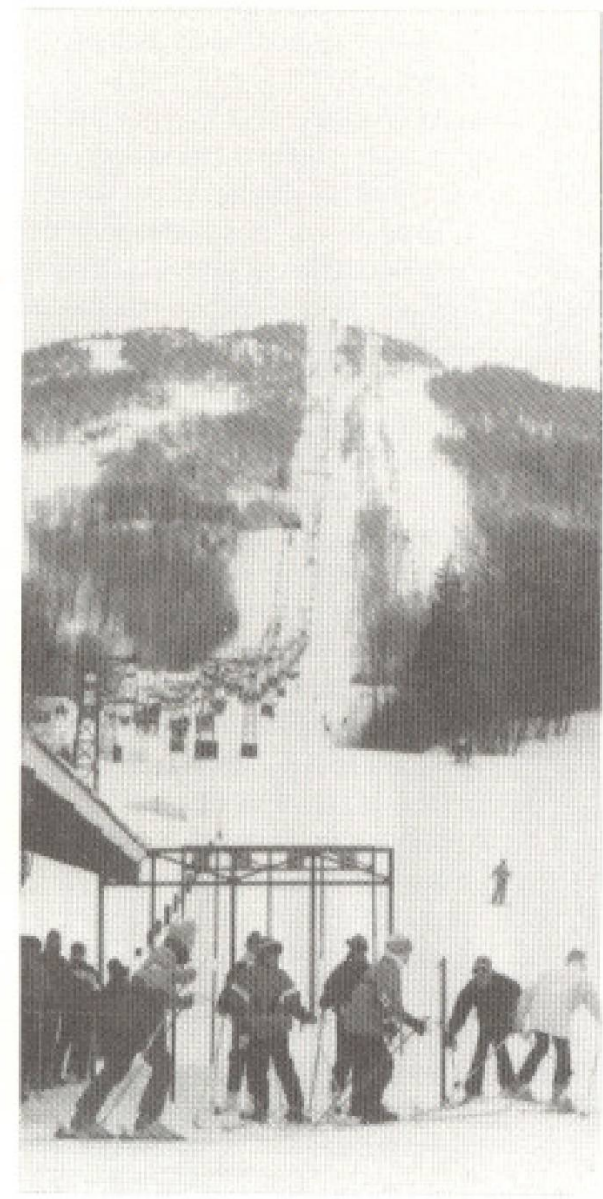

"Monsieur Fager Nadeau est Directeur du Departement de géographie at téledétection de l'Université de Sherbrooke et President de l'Association Touristique de I'Estrie (ATE|

\section{Présentation}

On a longtemps pris pour acquis que les vacances des Québécois se passaient presqu'essentiellement pendant la saison estivale; mais maintenant, on se rend de plus en plus compte que nous partons en vacances autant l'hiver que l'été (ou presque!). C'est bien souvent comment on vit - ou comment on perçoit le climat québécois qui détermine les destinations touristiques de la période hivernale. Pour certains, l'hiver québécois est à ce point difficile à supporter qu'ils en fuient les rigueurs en passant 1"hiver "dans le Sud", ce qui désigne habituellement la Floride, le Mexique ou les Antilles principalement. Pour $\mathrm{d}^{\dagger}$ autres, les premières neiges sont objet de réjouissances car on a depuis longtemps apprivoisé ce climat qui permet la pratique de plein d'activités stimulantes. Il y a enfin une troisième catégorie de personnes qui sont attirées et à l'aise dans les deux situations: ils vont chercher deux semaines de chaleur et dépaysement au Mexique ou en Martinique, en plus de passer deux ou trois mois sur leurs skis (alpins ou de randonnée). Bref, le climat, $c^{\text {test }}$ une question de goût, de perception, d'âge, de feeling, de mode, de marketing, de média. La Ville de Montréal, à elle seule, dépense $\$ 50$ millions pour faire disparaitre la neige de ses rues alors que les centres de ski dépensent des dizaines de millions pour répandre de la neige sur les pistes. Notre climat aux quatre saisons est bien "vivable" mais perçu différemment d'une personne à l'autre.

\section{A - L'appel du Sud}

Si les statistiques sur la fréquence des voyages intra-Québec ne sont guère reluisantes, ayant diminuées de $24 \%$ de 1980 à 1986, si la part du marché de la destination Québec dans les dépenses touristiques totales des Québécois est passée de $40 \%$ en 1980 à $34 \%$ en 1986 , si le tourisme représentait $2,6 \%$ du produit intếrieur brut (PIB) en 1986 comparativement à $3,2 \%$ en 1980, si notre balance des paiements, au niveau des échanges touristiques enregistrent, année après année, de nouveaux défi- cits records, c'est en partie due à nos pratiques hivernales.

Selon une recherche effectuée par l'hebdomadaire économique "Les Affaires" ${ }^{\text {"(2) }}$, entre novembre 1988 et avril 1989, au total, e'est environ 1228000 Québécois qui devaient quitter leur domicile pour prendre des vacances, auxquelles ils alloueraient un budget global de $\$ 1,740$ milliards. C'est presque autant que les vacances estivales.

Les auteurs de la recherche signalent qu'à l'heure actuelle, les Québécois dépensent plus de un milliard de dollars dans le Sud à chaque hiver et que cette tendance semble vouloir s'accentuer. Un profil similaire semble se dessiner en faveur des destinations européennes, non seulement en direction des zones de soleil mais aussi en faveur des stations de ski européennes.

Bien que le Mexique, la République Dominicaine, Cuba, et depuis peu, le Vénézuela et quelques pays d'Amérique Centrale attirent les Québécois par dizaines de milliers, c'est encore la Floride qui reçoit le plus de touristes Québécois l'hiver.

L'hiver dernier, les vacanciers Québécois ont dépensé dans les hôtels, restaurants et commerces de la Floride, près de $\$ 400$ millions. C'est du moins ce que révèle le premier numéro $\mathrm{d}^{+} u n e$ lettre circulaire, publiée à Orlando par le journal Le Soleil de la Floride, un mensuel de langue française qui tire à plus de 50000 exemplaires! Rien de surprenant puisque le ministère du Tourisme de la Floride estime à 578034 le nombre de touristes Québécois ayant séjourné dans l'État au cours de l'hiver 19881989. Ici aussi, on note une tendance à la hausse: de 1985 à 1988, les dépenses effectuées en Floride par les Québécois ont presque doublé, passant de $\$ 175$ millions à $\$ 390$ millions. Mais, ce n'est pas tout carle ministère du Tourisme prévoit que 700000 Québécois visiteront l'Etat l'hiver prochain, dépensant $\$ 600$ millions et générant à eux seuls des retombées économiques de l'ordre de $\$ 3$ milliards. Et dire que les Québécois 
"A y regarder de près, méme le climat. dont onditpourtant qu il est horrible, est plus tolerant que bien d'autres dont on dit qu ils sont merveilleux. La neige et le froid? Nous sommes equipes pour..

Les grandes catastrophes climanques nous sont a peu près inconnues. Les ouragans, les tomades, les tremblements de terre, lesinondations, les secheresses, qui font des ravages presque partout dans le monde, nous sont, la plupart du temps, epargnes.

Ce n'est pas la Cote d'Arur, j'en conviens, mais ce $n$ 'est pas non plus le Sahel de la sccheresse ou l'A menie aux 20 tremblements de terre par annee ou ces fles ou on n'en finit plus de se remettre du demier ouragan.

II y a l'hiver, bien suir. Trop long, bien sar. Mais amitons nous invente la mototheige ailleurs et connait-on ailleurs printemps plus eclatant?

Tolerant, notre climat, vous dis-je."o)

ne représentent que le quart des visiteurs en provenance du Canada! ${ }^{(j)}$

Oui, mais cela aussi, ça fait partie de notre réalité hivernale... Cela étant dit, allons maintenant jouer dans la neige québecoise pure laine!

\section{B - Le ski alpin}

\section{- 106 stations de ski}

Le Québec compte plus de 106 centres de ski, dont 9 sont reconnus comme des stations majeures; ces demières présentent une dénivelée qui atteint en moyenne 450 mètres (avec un maximum de 650 mètres à Mont Tremblant), ce qui les place légèrement derrière les principales stations du Vermont qui offrent des dénivelés variant de 600 à 1000 mètres.

Cette multiplicité de centres permet une grande diversité d'expériences car chacun de ces centres a sa personnalité propre. Par exemple, Mont Sainte-Anne accueille 650 000 jours/skieurs sur ses 43 pistes et sur ses trois versants; sa remontée à huit places et son village intégré aux pieds des pentes, le situe dans le clan sélect des Aspen, Whistler, Squaw Valley, Northstar et Stratton, selon la publicité(d). Par contre, dans les Appalaches gaspésiennes, le ski dans les
Chic=Chocs est héliporté; la dénivelée est de 850 mètres mais l'infrastructure est encore tellement faible que la capacité du centre n'est que de 20 skieurs héliportés par fin de semaine, ce qui donne un total de 230 jours/skieurs pour l'hiver 1988-1989(5). Quant à Bromont, cette station de vaste superficie, se caractérise par le fait que $40 \%$ de ses 280000 jours/ski soient en fait du ski de soirée ${ }^{(6)}$. Sur le massif de Petite-RivièreSt-François, dans Charlevoix, il n'y a pas de remonte-pente, pas de canon à neige malgré une dénivelée de 809 mètres, et on ne fait de ski que sur réservation et qu'avec un guide. Oui, en fait, l'autobus scolaire sert de remonte-pente et la capacité actuelle du centre est de 360 skieurs par jour sur 10 pistes. Il y a ici un projet de développement de $\$ 300$ millions qui vise à faire du Massif un centre international de sejour operant sur quatre saisons. Mais, le magouillage politique étouffe sa réalisation depuis une bonne décennie ${ }^{i 7}$. Terminons ici par le dessert: le Mont Tremblant, avec 650 mètres de dénivelée, 42 pistes et 540000 jours/ skieurs. Cequicaractérise Tremblant, c'est sans doute $1^{4}$ efficacité de sa mise en marche à l'extérieur du Québec: au moins la moitié de la clientèle de Mont Tremblant est américaine $^{(\mathrm{i})}$. Sutton et Gray Rocks sont aussi fort performants sur le marché américain.

Oui vraiment, pour les adeptes du ski alpin, le Québec peut répondre à toutes les attentes (ou presque...)

\section{-\$500 millions d'investissement (1983-1988) \\ (selon M. Henri Chapedeleine du MTO)}

Au début des années '80, la situation des stations de ski alpin au Québec n'était guère

"La technologie hivernale, c'est sans contredit le Québec qui la possède, comme la France possède la mode ou le vin, la Suisse, l'horlogerie, et 1'Allemagne, la stéréophonie: l'hiver. $c^{\prime}$ 'est à nous; $c^{7}$ est notre monde, $c^{7}$ est notre marque de commerce; la neige, c'est sûrement une de nos plus grandes richesses naturelles. Et comme toutes les autres, elle a besoin d'être exploitée. " $\times 9)$

(Laurent Beaudoin, Prés., Bombardier)

"L'hiver québécois représente pour nous et pour les étrangers un atout considérable au développement touristique et récréatif de la province. $^{\text {(1) }}(10)$

(Jacques Demers, Sous-ministre adjoint, MTO) reluisante: la vétusté de bien des équipements, le manque de liquidité pour procéder à des investissements majeurs, les problèmes liés à la sécurité des usagers, la vulnérabilité des centres face aux aléas de dame température et certains médias qui trouvent que c'est toujours mieux ailleurs, tout cela a contribué à détériorer la position concurrentielle du Québec comme destination touristique. Bref, cette industrie était sérieusement malade et même les skieurs québécois s'en donnaient de plus en plus à coeur joie dans les stations américaines.

C'est dans ce contexte pas très rigolo que le Gouvernement du Québec présentait, en 1983, son énoncé de politique sur le ski alpin $^{(11)}$. L'intervention gouvernementale avait comme objectif de redonneral' activite du ski alpin les atouts nécessaires pour rejoindre un plus grande nombre de skieurs (autant québécois qu'étrangers) et de susciter des retombées accrues. Des efforts très importants ont été consacrés à la réalisation de ces objectifs. On ne s'est pas contenté de faire des études mais Québec a montré son sérieux en mettant du "cash" sur la table, signe d'une véritable volonté politique (situation plutôt rare s'il en est une!). Et les résultats ont ếté extraordinaires. Cette réussite, grâce à l'implication concrète de Québec et à l'Entente CanadaQuébec sur le ski alpin, est probablement la plus remarquable qu'ait connu l'industrie touristique québécoise. Grâce à cette politique constructive, en cinq ans seulement, plus de $\$ 500$ millions ont été injectés dans le développement, la modernisation et l'infrastructure des stations, ainsi que dans l'amélioration de l'hébergement aux pieds des pentes. Au total, depuis 1982-1983, l'aide gouvernementale répartie sur 56 centres atteint près de $\$ 75$ millions, dont près de $75 \%$ proviennent du Québec et $25 \%$ du fédéral. Cette générosité n'est pas totalement désintéressée mais elle est de bon aloi; en 1986-1987 seulement, l'industrie du ski a généré des recettes fiscales de \$62 millions au niveau provincial et de $\$ 33$ millions au niveau fédéral ${ }^{(12)}$. Il est donc vrai de dire que c'est avec de $l^{*}$ argent que $l^{\text {'on }}$ fait de l'argent...

\section{0 de skieurs du Québec ${ }^{(13)}$ \\ 11560000 jours/ski en 1986-1987 $\$ 425$ millions de dépenses 10500 emplois-année}

La politique québecoise sur le ski alpin (1983) et les interventions financières des divers palliers de gouvernement ont été salutaires pour ce segment de l'industrie touristique qui a fait un bond en avant spectaculaire. Ainsi, en 1982-1983, nos 
stations enregistraient 1.9 millions de jours/ ski; cette donnée passait à 4.1 millions en 1985-1986 et 11.5 millions en 1986-1987. Selon les données de la firme CEGIR, la fréquentation des stations mesurée par le nombre de jours/skieurs s'est accrue en moyenne de $25 \%$ par année entre 1983 et 1987 , ce qui est absolument remarquable si on tient compte du fait qu'au même moment, partout en Amérique du Nord, la fréquentation avait plutôt tendanceàplafonner ${ }^{(14)}$. Pendant cette même période, le taux de participation de la population adulte du Québec à l'activité du ski alpin est passée de $7,5 \%$ à $15 \%$. De plus, l'intensité de la pratique s'est elle aussi accrue: elle était de 8,9 jours durant la saison d'hiver 1984 1985; elle est passéeà 10,1 jours/ski l'année suivante et à 11,4 jours/ski en 1986-1987(15) Spectaculaire, vous disais-je!...

En 1986-1987, \$425 millions ont été associés à la pratique su ski alpin au Québec et près des 3/4 de ces dépenses l'ont été par des "touristes", à l'occasion de voyages de 24 heures ou plus au Québec, lesquels impliquent le recours à de l'hébergement, commercial ou privé. Il va de soi que ce sont les Québécois eux-mêmes qui sont les principaux touristes dans les stations. Sur les \$425 millions dépensés au Québec, \$196 millions demeurent dans les centres de ski proprement dit tandis que $\$ 150,4$ millions vont dans I'hôtellerie et la restauration, $\$ 50,6$ millions à $1^{\prime}$ equipement et $\$ 28$ millions pour les vêttements.

Il est sans doute pertinent de signaler ici que sur les 11,5 millions jours/ski pratiqués au Québec, un million de jours/ski sont attribuables à des skieurs non Québécois. Pris globalement, les skieurs québécois, ontariens et américains dépensent respectivement $\$ 47, \$ 73$ et $\$ 108$ par jour, sans compter le transport. Ajoutons aussi que les skieurs québécois dépensent approximativement $\$ 100$ millions pour la pratique du ski à l'extérieur du Québec, ce qui représente près de $19 \%$ de toutes leurs dépenses. Au total, les Québécois ont dépensé plus de $\$ 511$ millions pour faire du ski en 1986-1987.

\section{- Des forces mais aussi des faiblesses}

Aux yeux des touristes, les principaux avantages comparatifs du produit skiquébécois seraient la qualité et la quantité de la neige en premier lieu. Au cours des dernières années, la plupart des principaux centres ont investi surtout dans le système d'enneigement artificiel, ce qui permet de couvrir jusqu'à $90 \%$ de la surface des pentes. L'expérience a montré que les systè- mes d'enneigement artificiel prolonge en moyenne de 15 jours la saison de ski, ce qui est bougrement important, puisque dans la majorité des cas, un centre a peu de chance de se rentabiliser à moins de 100 jours d'activité par année. Ajoutons que le nombre d'heures d'ouverture a augmenté de $34 \%$ (16).

Une autre partie importante des récents investissements visait à réduire le temps d'attente au bas des pentes. On retrouve maintenant un télésiège quadruple détachable dans plusieurs centres, ce qui accroît de beaucoup la capacité de remontée des skieurs. La qualité des écoles de ski, la restauration, l'ambiance et les prix sont aussi des atouts recherchés. Et puis, l'hébergement au pied des pentes, qui a longtemps été un obstacle au développement, a fait des progrès très considérable, spécialement à Mont Ste-Anne, Stoneham, Sutton, Owl's Head, Gray Rocks à Mont Tremblant et à Orford. "La région du Lac Memphrémagog a par exemple bénéficié d'une vêritable explosion de son réseau régional d'hébergement. Depuis 1984, plus de $\$ 100$ millions ont été investis en hébergement locatif dans un rayon de $7 \mathrm{~km}$ de la station du Mont Orford"(in).

Bref, dans l'ensemble, le produit s'est considérablement amélioré... mais ce n'est pas encore la maturité. En effet, il reste encore des efforts importants à faire. Par exemple, de façon générale, les stations destination du Québec accusent un retard sur leurs rivales de la Nouvelle-Angleterre en ce qui a trait au réceptif et à la commercialisation. Les budgets accordés au marketing sont relativement moins élevés et l'organisation des activités de commercialisation est moins avancée au Québec ${ }^{(12)}$. La faible dénivellation des pentes, l'éloignement, la temperature incertaine, les files d'attentes, le manque de confort et le choix d'activités complémentaires sont aussi considérés comme des faiblesses par les touristes skieurs. À cela, il faut ajouter que le marché local des skieurs approche de son point de saturation (si ce n'est déjà fait) et que la fréquentation des centres est beaucoup trop concentrée sur les fins de semaine: à l'heure actuelle, $60 \%$ des jours/ ski sont concentrés sur les deux journées de fin de semaine. Le marché touristique présente un énome potentiel mais il devient de plus en plus compétitif et le développement d'activités en dehors de la saison de ski demeure l'apanage d'un nombre encore trop limité de stations. Finalement, il faut signalerque la situation financière de plusieurs stations a de quoi inquiéter.

\section{- Perspectives d'avenir}

Il nous apparaît que ce segment important de l'industrie touristique du Quebec a un avenir pour le moins incertain. Bienque les gouvernements provincial et fédéral retirent des recettes fiscales de plus de $\$ 100$ millions par année liées à l'activité ski alpin, ils ont récemment mis fin à l'entente Canada/ Québec qui fut dans une large mesure responsable du développement des cinq dernières années. Ces "coupures" pourraient avoir des conséquences fort sérieuses et ces "économies" à courtes vues pourraient coûter très chères.

Il est à prévoir que le retrait de l'aide gouvernementale obligera les gestionnaires des stations à hausser rapidement le prix des billets de ski qui rejoindra progressivement la tarification américaine, soit autour de $\$ 35$ U.S. par jour. Le Québec perdra ainsi l'avantage concurrentiel qu'il détenait et il $y$ a fort à parier que le nombre de Québécois allant skier aux États-Unis augmentera substantiellement. On a signalé précédemment que, suite aux investissements massifs des dernières années, plusieurs stations sont en manque de liquidités et qu'avec, le retrait de l'aide gouvernementale ralentira les nouveaux investissements, les stations concurrentes de Nouvelle-Angleterre prendront une avance technologique sur celles du Québec. L'augmentation des coutts pour le consommateur, le ralentissement du développement des stations et le vieillissement rapide de la population risquent d'entraîner un recul de l'activité au Québec d'ici cinq ans.

Selon l'étude de CEGIR, du million de skieurs actifs au Québec, les 3/4 ont moins de 35 ans. Le vieillissement progressif de notre population devrait entraîner une réduction significative du nombre de pratiquants et donc de la fréquentation des centres. Bien sûr, on peut mener des campagnes de publicité pour inciter les gens de plus de 35 ans à pratiquer davantage le ski mais les quelques gains que l'on pourra faire seront anéantis par les pertes résultant de l'augmentation des prix. Car il y a des limites à tout. Il est utile de se rappelerqu'il $y$ a quelques années à peine, le billet journalier dans un centre de ski était de $\$ 10$ à $\$ 12$; il atteint aujourd "hui de $\$ 25$ a $\$ 30$ et il dépassera probablement $\$ 35$ dans $2-3$ ans. sans compter la TPS. Mais le prix du billet, ce n'est pas tout. Le coût de l'équipement (bottes, ski et fixations) a plus que doublé depuis 1985. Et un equipement bas de gamme coûte aujourd'hui $\$ 300$ à $\$ 450$... mais qui veut en ces jours d'un equipement. bas de gamme? Il n'en reste pas moins 


\section{IMPACT D'UN CENTRE DE SKI; CAS: STATION DU MONT ORFORD}

En opération depuis 1938.

3 massifs et 4 versants repartis sur les monts Giroux, Orford et Alfred Destochers.

853 metres d'altitude.

540 metres de dénivellation.

Capacité de 11400 skieurs a I heure sur 31 pistes.

enneigement artificiel sur $85 \%$ du domaine skrables.

Nombre de jours/ski 1984-85: 160000

Nombre de jours/ski 1988-89:360000

Nombre de places d'hebergenent: (lits) a vocation locative en 80-81: 717 dans un rayon de $7 \mathrm{~km}$. en 88-89: 5000 lits.

Investissement deptis 1984 a la Station et au Village Mont Orford: 26 millions de dollars.

Investissement régional en hébergement dans la région Magog-Orford depuis 1984: ples de 100 millions de dollars.

Nombre d'employés a la station de ski durant la saison 79-80: 95 - durant la satson 88-89: 320 .

Supposons 320 personnes travaillant pendant 12 semaines, $40 \mathrm{hrs}$ a $8.50 \mathrm{st}$ hir. ca ferait 1305600 S en salaire ...

Mont Orford a remporté le "Flocon d $\mathrm{Or}^{\text {"; }}$ déceme par le réseau info-ski MRG comme station renouvelée de l'année 1988. La station a aussi éte finaliste du Mercure pour I'entreprise de service de 1988, en plus de remporter le Grand Prix di tourisme régional et NATIONAL, categonie "developpement", 1988. (Accueil R en 1989).

Orford recevra le Championnat canadien do ski, du 14 au 17 fevrier 1990 et le championnat Inter-Continental Universitaire, du 18 au 20 mars 1990.

\section{Sources: Pierrette Rivest \\ Michel Lafleur \\ Mont Orford}

une vraie saison touristique et par l'adoption de stratégies de commercialisation et de marketing plus professionnelles.

Poursuivre le développement et l'intégration entrepris, ça peut vouloir dire de rester à la fine pointe du développement technologique, de continuer à développer un hébergement et une restauration de qualité au pied des pentes, sans oublier toute la gamme des activités d'animation et de récréation susceptibles de rendre le séjour plus agréable - ou qui donnent envie de prolonger le séjour. Ça peut aussi vouloir dire pour plusieurs stations de penser leur développement surquatre saisons, facilitant ainsi l'amortissement des coûts sur 300 jours au lieu de 100 . Poursuivre en ce sens, c'est s'assurer de conserver chez nous $85 \%$ à $90 \%$ de la clientèle québécoise. Cette clientèle de base est essentielle mais elle est insuffisante pour justifier un développement important. Il faut donc aller chercher une part plus large de clientèle hors-Québec.

A l'heure actuelle, environ $10 \%$ de la clientèle de nos stations de ski provient de l'extérieur du Québec. Les skieurs touristes quant à eux proviennent du Québec à $63 \%$, de l'Ontario $25 \%$ et des USA a $12 \%$. Ces derniers pourcentages devront être augmentés. Les États-Unis comptent de 7 à 8 millions de skieurs actifs, lesquels ont réalise 53,7 millions de jours/ski en 19861987. Quant à 1'Ontario, elle compte 1,4 millions de skieurs qui pratiquent cette activité en moyenne 10,4 fois par saison. Un des objectifs prioritaires du ski québécois devrait être de se doter des outils de marketing, de mise en marché et de commercialisation lui permettant de doubler, sinon tripler, sa part de clientèle sur le marché américain et ontarien. Cette clientèle a l'avantage de présenter une durée de séjour variant entre 4 et 5 jours par voyage. Mais, il semble bien que des segments entiers de ce lucratif marchể ne soient à peu près pas sollicités. Les plus gros clubs de ski aux USA se trouvent à Miami et à New York; ces deux clubs comptent jusqu'à 35000 membres. Le Mont Tremblant est à peu près le seul à exploiter ce marché à fond en préparant des forfaits tout à fait adaptés à cette clientèle. A Mont Tremblant, les résultats sont probants. On pourrait en faire autant ailleurs si on utilisait la méme approche professionnelle. Autre exemple: Gray Rocks. Ici, la montagne ne casse rien mais c'est tout de même plein d'Américains tout

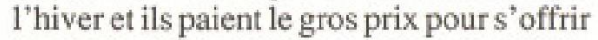
l'ambiance Gray Rocks. Ici, on mise sur une commercialisation professionnelle de tous les instants qui cherche à combler le skieur adulte. On ne mise pas uniquement 
sur le produit ski mais également sur un ensemble d'éléments incluant le ski. Ainsi, la station n'est pas prise au dépourvu si les conditions de ski ne sont pas adéquates car les facilités et les infrastructures d'accueil, d'hébergement, de restauration et $\mathrm{d}^{\prime}$ animation sont ajustés en conséquence ${ }^{(12)}$. Bien des stations pourraient s'améliorer en ce sens.

Par exemple, on a toujours dit que "ltaprèsski" était très faible au Québec, souvent inexistant. Pourtant, peu a été fait pour corriger cet état de fait, sauf pour les stations situées à proximité de centre urbain, comme Ste-Anne, St-Sauveur ou Orford. Autre exemple: la majorité des centres fonctionnent a $125 \%$ en fin de semaine... et à $25 \%$ pendant la semaine. Pour favoriser un meilleur équilibre, il faudra introduire une tarification des billets de remontée plus différencié. Si le tarif journalier en fin de semaine est de $\$ 25$ par personne, si on veut que ce tarif différencié soit vraiment un incitatif à skier sur semaine, celui-ci devrait se retrouver entre $\$ 15$ et $\$ 19$. Trop de gestionnaires offrent un rabais d'un dollar ou deux et s'étonnent de ne pas voir d'impact...! Signalons cependant que Bromont et le Relais de St-Castin notamment, après avoir tenté diverses expériences à cet égard, en ont conclu que la différenciation des prix n'avait aucun impact sur leur clientèle. Bien d'autres cas pourraient être soulevés mais enfin, rappelons qu'à ce chapitre (marketing-commercialisation) il y a tellement de choses qui restent à faire. Entre 1984 et 1987 , le nombre de skieurs-touristes a doublé au Québec. Si on le veut vraiment, on peut encore doubler ce nombre d'ici 3 à 5 ans. La qualité et le professionnalisme se vendront toujours bien.

L'industrie a le choix des scénarios. Quant à l'État, il a aussi le choix; ou bien il se donne le mandat d'aider l'industrie à atteindre des sommets dont tous profiteront, ou bien il se spécialise dans les coupures paralysantes... On sait qu'il peut être très efficace dans les deux situations...!

\section{Notes explicatives}

(1) BOUnGault, Pierre, Mol, je m'en souviens, Editions Stanke. Montréal, 1969, pp. 150-151.

(2) HËBERT, Diane, Vacances d'hiver; les Ouebecois deipenseront \$1, 74 miliards cet hiver. Les Affaires, vol. $L X$, no 48, 3-9 décembre 1988, Montreal, pp. 25

(3) GIRARD, Maurice, 7000000 Ouébécois se rendrant en Floride l'hiver prochain. Presse canedienne ide Washington), La Presse, Montréal, edition du 31 juillet 1989 , A-5.

(4) BOURCIER, Gilles, Mont Sainte-Anne: plus gros. plus riche, plus skiable La Presse, Montreal, édition du 22 féwrier 1989 ,

(5) BOURCIER, Gilles, Chic-Chocs: tout ce potentiel inexploitel, La Presse, Montreal, édition du 6 avril 1989.

(6) BOURICIER, Gilles, Bromont: pour le ski de soiree et le perty, La Presse, Montreal, édition du 29 mars 1989.

(7) BOURCIER, Gilles, Lemassif, La Presse, Montréal, edition du 15 fávrier 1989

(8) BOURCIER, Gilles, Mont Tremblant: la Mecque du ski laurentien, La Presse, Montréal, édition du 22 mars 1989.

(9) BEAUDOIN, Laurent, in NADEAU et Al.. publication de I'ATT \#10: Les journèes d'information sur le tourisme hivernal au Quebec, rapport des réunions tenues à Sherbrooke le 1eret 2 décembre 1978, pp. 14-15,

(10) DEMERS, Jacques, Ibid., p. 76 .

(11) MLCP, La politique québécoise pour le ski alpin, gouvernement du Quétec, Quebec, octobre 1983.

(12) CEGIA, L'industrie du ski alpin au Québec: bilan et horizons - Rapport final, entente Buxiliaire Canada-Québec sur le développement touristique, Montréal, février 198 /pagination specialel.

113) CLOUTIEA, Laurier, Le tarif des remonte-pentes condamné aे grimper, La Presse, edition du 7 janvier 1989.

114) LES AFFAIRES, no 43, wolume LX, Montreal, 29 octobre-4 nowembre 1988 .

(15) CLUZEAU, Patrick, Le tourisme hivernal des Québécois durant la saison d'hiver 1986/87. Les skieurs alpin, les motoneigistes, les skieurs de randonnée, Gouvernement du Québec. ministère du Tourisme, direction de l'Analyse et du Développement, Cuébec, mars 1988, 188 pages.

(16) CHAPDELAINE. Henri, conférence donnée au Colloque sur le tourisme hivernal, UQAM, 22 fortier 1989.

(17) DUBUISSON, Philippe, L'hibergement insuffisant nuit a la craissance de plusieurs centres de ski, Les Affaires, Montréal, 29 octobre 1988.

(18) DUPAUL, Richard, Le ski en pente douce? Lo Presse, édition du 15 février 1989. Voir aussi Voyages d'aftaires et vacances d'hiver, in cahier spécial Les Affaires, montráal, 14 oçtobre 1989 16 pages. 